\title{
Cluster Algorithm Renormalization Group Method
}

\author{
Guillermo Palma* and David Zambrano \\ Departamento de Física \\ Universidad de Santiago de Chile \\ Chile. \\ E-mail: guillermo.palma@usach.cl
}

We present a self consistent method based on cluster algorithms and Renormalization Group on the lattice to study critical systems numerically. We illustrate it by means of the 2D Ising model. We compute the critical exponents $v$ and $\eta$ and the renormalization group flow of the probability density function of the magnetization. The results, compared to the standard Monte Carlo Renormalization Group proposed by Swendsen [1], are very accurate and the method works faster by a factor which grows monotonically with the lattice size. This allows to simulate larger lattices in reachable computational times.

The XXVI International Symposium on Lattice Field Theory

July 14-19 2008

Williamsburg, Virginia, USA

\footnotetext{
*Speaker.
} 


\section{The Method}

We consider a general spin model defined in a square lattice of lattice spacing $a$, and linear size $L$, with periodic boundary conditions which is defined by the Hamiltonian

$$
H=\sum_{\alpha} K_{\alpha} S_{\alpha}
$$

where each $S_{\alpha}$ is a combination of the spin variables, for example $S_{1}=\sigma_{i} \sigma_{j}$ for first neighbors, $S_{2}=\sigma_{l} \sigma_{m}$ for second neighbors, and so on.

In this article we illustrate the method by considering the 2D Ising model, including up to three even interactions (nearest-neighbor, second-neighbor, and four spin) and one odd interaction (a weak magnetic field).

The RG theory allows the computation of the critical properties of a model. The critical exponents for example, can be obtained from the linearized RG transformation matrix $T_{\alpha \beta}{ }^{*}$, by computing its eigenvalues. $T_{\alpha \beta}{ }^{*}$, defined by

$$
T_{\alpha \beta}{ }^{*}=\left[\frac{\partial K_{\alpha}^{(n)}}{\partial K_{\beta}^{(n-1)}}\right]_{H^{*}}
$$

can be obtained numerically from the coupled equations,

$$
\frac{\partial\left\langle S_{\gamma}^{(n)}\right\rangle}{\partial K_{\beta}^{(n-1)}}=\sum_{\alpha} \frac{\partial K_{\alpha}^{(n)}}{\partial K_{\beta}^{(n-1)}} \frac{\partial\left\langle S_{\gamma}^{(n)}\right\rangle}{\partial K_{\alpha}^{(n)}}
$$

where,

$$
\frac{\partial\left\langle S_{\gamma}{ }^{(n)}\right\rangle}{\partial K_{\beta}{ }^{(n-1)}}=-\left\langle S_{\gamma}^{(n)} S_{\beta}^{(n-1)}\right\rangle+\left\langle S_{\gamma}^{(n)}\right\rangle\left\langle S_{\beta}^{(n-1)}\right\rangle
$$

and

$$
\frac{\partial\left\langle S_{\gamma}^{(n)}\right\rangle}{\partial K_{\alpha}^{(n)}}=-\left\langle S_{\gamma}^{(n)} S_{\alpha}^{(n)}\right\rangle+\left\langle S_{\gamma}^{(n)}\right\rangle\left\langle S_{\alpha}^{(n)}\right\rangle
$$

The critical exponents are obtained from the eigenvalues of $T_{\alpha \beta}{ }^{*}$ in the standard way. For the Ising model they are given by the standard relations $v=\ln s / \ln \lambda_{1}{ }^{e}$ and $\eta=d+2-2 \ln \lambda_{1}{ }^{o} / \ln b$, where $\lambda_{1}{ }^{e(o)}$ is the largest even (odd) eigenvalue.

\section{The CARG method}

For the 2D Ising model the Hamiltonian reads

$$
H(\sigma)=\frac{J}{k_{B} T} \sum_{<i, j>} \sigma_{i} \sigma_{j}
$$

where $J>0$ describes a ferromagnetic system. In the thermodynamic limit this model has a second order phase transition at the Onsager's critical temperature $T_{c}=2 / \ln (1+\sqrt{(2)})$. 


\subsection{Magnetic Susceptibility}

In order to compute the linearized RG transformation matrix $T_{\alpha \beta}{ }^{*}$ from eqn. (1.2), the critical temperature should be used. One can obtain it by computing the susceptibility for different lattice sizes as shown in figure 1. Its maximum for each lattice size $L$ defines the lattice shifted critical temperature $T_{c}(L)$.

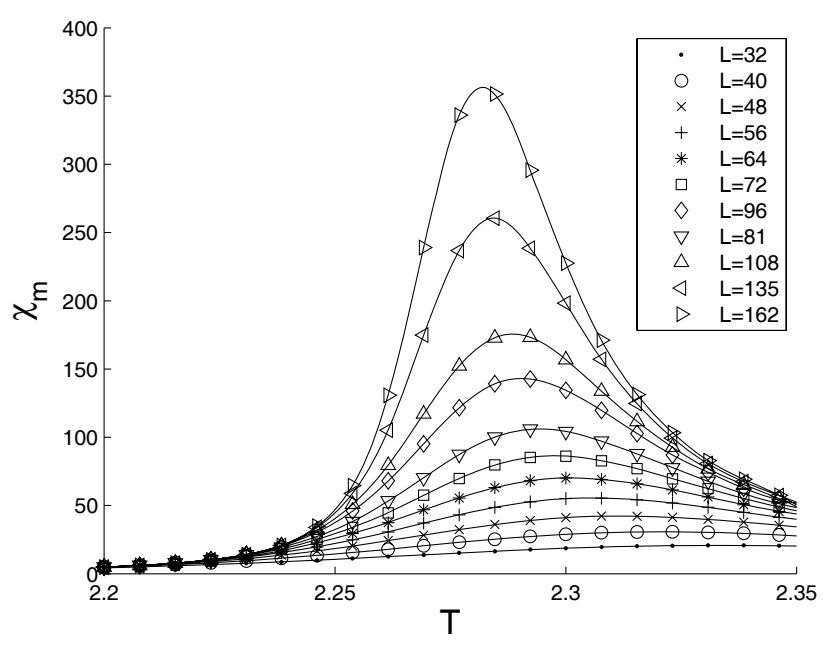

Figure 1: The maximum of the susceptibility $\chi_{m}[L]$ is obtained using interpolation. For example, $\max \left(\chi_{m}\right)[L=108]=2.2886, \max \left(\chi_{m}\right)[L=64]=2.3008$, and $\max \left(\chi_{m}\right)[L=45]=2.314$.

\subsection{Finite Size Scaling}

Now we perform a finite size scaling analysis (FSS) of the numerical results obtained above to extract the infinite volume limit of the critical temperature. We use the standard analytical formula $T_{c}(L)=T_{c}(\infty)+\alpha / L$ to fit the numerical values $T_{c}(L)$. The fit is shown in figure 2 .

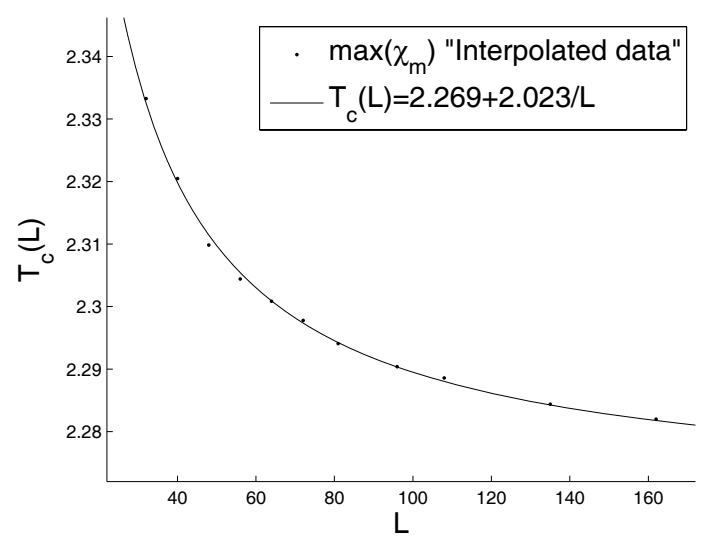

Figure 2: From the fit we obtain the numerical values $T_{c}(\infty)=2.269$, and $\alpha=2.023$. The convergence to $T_{c}(\infty)$ is fairly fast, as can be seen from the following values for the lattice shifted critical temperature: $T_{c}(L=108)=2.2877, T_{c}(L=64)=2.3006, T_{c}(L=45)=2.3140$. 
The thermodynamic limit of the lattice shifted critical temperature $T_{c}(\infty)$ agrees with the Onsager's critical value up to an error less than 0.8 per one thousend. At this point we want to emphasize that a precise value for exact value of the critical temperature is not necessary to compute the eigenvalues of the linearized RG transformation matrix. In fact, it is only necessary to calculate the derivatives appearing in eqn. (1.2) in a neighborhood of a "linear region", where they are essentially constant.

\subsection{Binder Cumulant}

One alternative method to compute the critical temperature of the infinite volume system is to use the Binder Cumulant

$$
u(L, T)=1-\frac{1}{3} \frac{\left\langle M^{4}\right\rangle}{\left\langle M^{2}\right\rangle^{2}} .
$$

which becomes independent of the lattice size at the Onsager's critical temperature. This behavior is shown in figure 3 .

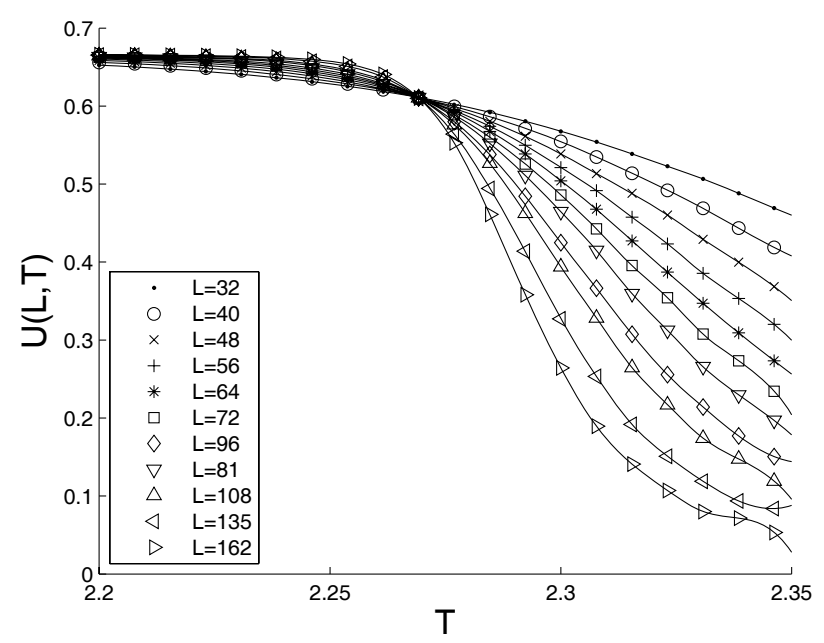

Figure 3: The intersection of these curves occurs at: $T_{c}(\infty)=2.26903 \pm 0.00059$. This value differs in 1 percent from Onsager's exact result.

The result obtained with the Binder Cumulant is consistent with the above result obtained by a FSS analysis.

\section{Numerical Results}

\subsection{Computing the critical exponents.}

To perform the simulation on the fundamental lattice we used the Wolff algorithm [2]. A total of $10^{5}$ sweeps were used to thermalize the system and $10^{6}$ configurations were used to compute thermal averages. Up to three even interactions (nearest-neighbor, second-neighbor and four spin or plaquette) and one odd interaction (magnetic field) were considered. We considered a lattice of lattice size $L=64$ and performed two RG steps and used the renormalization parameter $b=2$. The 
results for the critical exponents $v$ and $\eta$ are displayed in the following table. Compared to the exact values, the numerical results agree remarkably well, with errors of one in one thousand.

\begin{tabular}{|c||c|c|c|c|}
\hline \hline RG Step & $\lambda_{1}{ }^{e}$ & $\lambda_{1}{ }^{o}$ & $v$ & $\eta$ \\
\hline 1 & 1.9586 & 3.6856 & 1.0311 & 0.2362 \\
\hline 2 & 1.9986 & 3.6676 & 1.0010 & 0.2503 \\
\hline Exact & 2 & 3.6680 & 1 & 0.250 \\
\hline \hline
\end{tabular}

Table 1: Critical exponents for $L=64$ and $T=2.259$.

In order to compare our results with the ones obtained in reference [3], we considered a lattice of lattice size $L=108$ and performed three RG steps using the renormalization parameter $b=3$. The results are shown in the following table, where the label [S] stands for the result of ref. [3] and [PZ] for ours.

\begin{tabular}{|c||c|c|c|c|}
\hline \hline RG Step & $\lambda_{1}{ }^{e}$ & $\lambda_{1}{ }^{o}$ & $v$ & $\eta$ \\
\hline $1_{[S]}$ & 2.852 & 7.705 & 1.048 & 0.2828 \\
\hline $1_{[P Z]}$ & 2.8635 & 7.7062 & 1.0443 & 0.2825 \\
\hline $2_{[S]}$ & 3.021 & 7.828 & 0.994 & 0.2540 \\
\hline $2_{[P Z]}$ & 3.0027 & 7.8269 & 0.9992 & 0.2542 \\
\hline $3_{[S]}$ & 3.007 & 7.831 & 0.998 & 0.2534 \\
\hline $3_{[P Z]}$ & 3.0013 & 7.8361 & 0.9996 & 0.2521 \\
\hline Exact & 3 & 7.8452 & 1 & 0.250 \\
\hline \hline
\end{tabular}

Table 2: The critical exponents obtained by the CARG method [PZ] and by Swendsen's method [S] for $L=108$ are displayed.

The numerical results for the critical exponents $v$ and $\eta$ are very accurate and both results ([S] and [PZ]) agree remarkable well with their corresponding exact values. The main advantage of our method consists that it is faster than the method proposed by [3] by a factor which increases with the system size.

\section{RG-flow of the PDF}

\subsection{Probability Density Function}

As it was pointed out in [4], the probability density function (PDF) of the magnetization corresponds to the partition function of an auxiliary theory, which equals the original theory plus a very small imaginary coupling. From RG theory one knows that partition functions are invariant under $\mathrm{RG}$ transformations. This is confirmed directly in the following figure

The first plot of figure 4 shows the PDF for the fundamental lattice and for two coarse grained lattices. In the second plot the PDFs normalized to the first two moments are displayed. The three curves collapse onto one curve with high accuracy, showing the scale invariance of the PDF. 

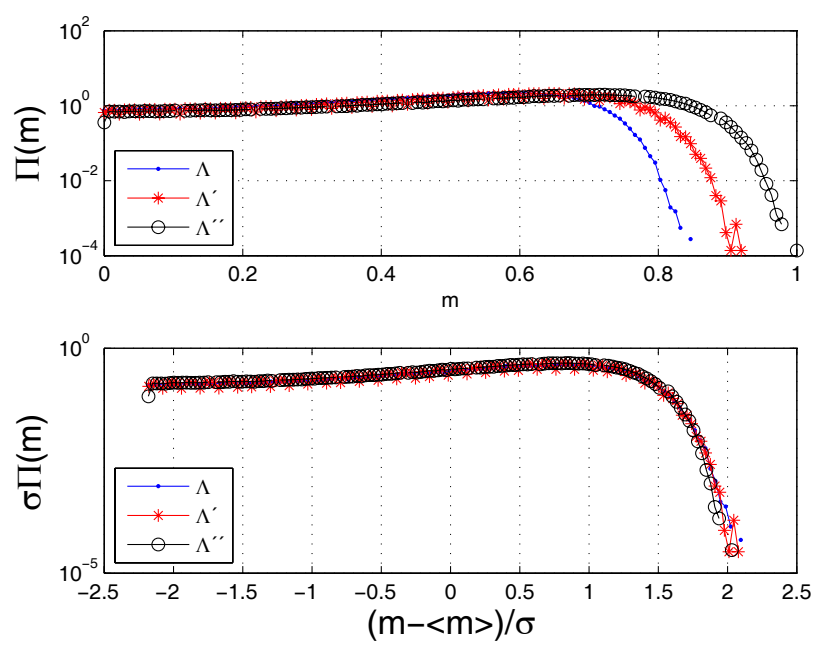

Figure 4: $L=64$ at $T=T_{c}(L)=2.3008 . \Lambda \rightarrow L=64 . \Lambda^{\prime} \rightarrow L=32 . \Lambda^{\prime \prime} \rightarrow L=16$.

\section{Conclusions}

We have shown how to obtain very accurate values for the critical exponents and how to compute the RG flow of the PDF of the magnetization without the previous knowledge of the critical temperature. The method is self consistent and when compared to the original method proposed in [3], our method is faster by a factor which grows linearly with the lattice size from 6 for $L=64$ until a factor 10 for $L=162$. The use of a cluster algorithm to simulate the fundamental Hamiltonian leads to this advantage, which allows to simulate larger lattices in reasonable computation times. Further application of the method in connection with universal fluctuations will be reported elsewhere [5].

\section{Acknowledgments}

This work was partially supported by DICYT of the University of Santiago de Chile.

\section{References}

[1] R. H. Swendsen, Phys. Rev. Lett., 42, (1979) 859.

[2] U. Wolff, Phys. Rev. Lett., 62, (1989) 361.

[3] R. H. Swendsen, Phys. Rev. B, 20, (1979) 2080-2087.

[4] G. Mack, G. Palma and L. Vergara, Phys. Rev. E, 72026119 (2005).

[5] G. Palma and D. Zambrano, submitted to Phys. Rev. E (2008). 